\title{
Molecular basis of phosphoryl guanidine oligonucleotides elongation by Taq DNA polymerase
}

\author{
Alexander Lomzov \\ ICBFM SB RAS, Novosibirsk, Russia \\ lomzov@niboch.nsc.ru
}

\author{
Dmitrii Pyshnyi \\ ICBFM SB RAS, Novosibirsk, Russia \\ pyshnyi@niboch.nsc.ru
}

\begin{abstract}
Phosphoryl guanidine oligonucleotides (PGO) become essential tools for basic research in molecular biology, molecular diagnostics and perspective for the therapeutics. To determine molecular basis of the observed effects of PG modification on elongation efficiency we performed molecular dynamics simulation of Taq polymerase / PGO complexes. The data obtained shows most important points for the analysis of protein / PGO interaction and gives useful information for development of improved PCR analysis.
\end{abstract}

Keywords - Phosphoryl guanidine oligonucleotides, Taq polymerase, molecular dynamics

Motivation and aim

Motivation

Phosphoryl guanidine oligonucleotides (PGO) become essential tools for basic research in molecular biology, molecular diagnostics and perspective for the therapeutics [14]. A number of problems in PCR diagnostics could be solved by the using of PGOs. The influence of phosphoryl guanidine (PG) group(s) on elongation of primers by Taq DNA polymerase were studied experimentally. To determine molecular basis of the observed effects we analyzes of Taq polymerase / DNA complexes using computational approaches.

Aim

To determine molecular basis of the observed effects of PG modification on elongation efficiency we performed molecular dynamics simulation of Taq polymerase / PGO complexes.

\section{Methods}

The complex was build based on the structures PDBIDs: $1 \mathrm{TAQ}$ and 5E41. Lacking fragments of protein were added in disordered form.

Classical full atomic molecular dynamics simulations in implicit solvent water model were performed using Amber software, ff99bsc1 force field for DNA and ff14SB - for protein. The data obtained was analyzed using ccptraj and UCSF Chimera software.

\section{Results}

A number of complexes Taq polymerase and native and PG-containing oligonucleotides were analyzed. All complexes were stable along MD trajectory $(50-100 \mathrm{~ns})$. Most important PG positions of different diastereomers which are critical for PGO elongation were determined. The data obtained in good agreement with the experimental results. The results of the study shows most important points for the analysis of protein / PGO interaction and gives useful information for development of improved PCR analysis.

\section{ACKNOWLEDGMENT}

Supported by the RSF (18-14-0035)7 and by State project No. A-0309-2016-0004.

\section{REFERENCES}

[1] Kupryushkin, M. S., Pyshnyi, D. V., Stetsenko, D. A. (2014). Phosphoryl guanidines: a new type of nucleic acid analogues. Acta Naturae, 6(4): 116-118.

[2] Garafutdinov, R. R., Sakhabutdinova, A. R., Kupryushkin, M. S., \& Pyshnyi, D. V. (2020). Prevention of DNA multimerization using phosphoryl guanidine primers during isothermal amplification with Bst exo-DNA polymerase. Biochimie. 168: 259-267.

[3] Lomzov, A. A., Kupryushkin, M. S., Shernyukov, A. V., Nekrasov, M. D., Dovydenko, I. S., Stetsenko, D. A., Pyshnyi, D. V. (2019). Diastereomers of a mono-substituted phosphoryl guanidine trideoxyribonucleotide: isolation and properties. Biochemical and biophysical research communications. 513(4): 807-811.

[4] Kuznetsov, N. A., Lebedeva, N. A., Kuznetsova, A. A., Rechkunova, N. I., Dyrkheeva, N. S., Kupryushkin, M. S.,... \& Lavrik, O. I. (2017). Pre-steady state kinetics of DNA binding and abasic site hydrolysis by tyrosyl-DNA phosphodiesterase 1. Journal of Biomolecular Structure and Dynamics. 35(11): 2314-2327. 